EDEECONOMIAE
DEGRONEGÓCIO

ISSN impresso: 1679-1614

ISSN online: 2526-5539

Vol. 17 | N. 3 | 2019

Mirian Oliveira de Souza ${ }^{1^{*}}$

ORCID: $0000-0002-3710-3957$

Mirian R. Piedade Bacchi ${ }^{2}$

ORCID: $0000-0001-9413-8614$

Lucilio Rogerio A. Alves ${ }^{2}$

ORCID: 0000-0002-9959-590X

1 Empresa Brasileira de Pesquisa Agropecuária - Embrapa, Brasília,

Distrito Federal, Brasil.

2 Escola Superior de Agricultura "Luiz de Queiroz", Universidade de São Paulo, Piracicaba, São Paulo,

Brasil.

* mirian.souza@embrapa.br

Recebido em: $18 / 12 / 2018$

Aceito em: 08/07/2019

\section{ANÁLISE DE FATORES QUE INFLUENCIAM O PROCESSAMENTO DE SOJA NO BRASIL}

\section{RESUMO}

Este artigo analisa os fatores que influenciam o processamento doméstico de soja, com dados de séries mensais de 2005 a 2015. Para tanto, desenvolve-se e ajusta-se um modelo teórico cujas hipóteses são testadas por meio de Modelos de Autorregressão Vetorial com Correção de Erros (VEC). As informações obtidas com a observação desses fatores complementam a literatura existente sobre o mercado de soja, a qual é, geralmente, voltada à análise da relação entre preços. Os resultados indicam que, entre as variáveis consideradas no modelo, a renda é a que apresenta maior influência sobre o processamento doméstico de soja, com efeito positivo e mais do que proporcional, sugerindo que a demanda por produtos processados aumenta quando o poder de compra cresce. A renda também impacta forte e positivamente as quantidades abatidas de suínos e de aves, indicando que à medida que o poder de compra aumenta, o consumo de carnes tende a aumentar. De modo geral, o modelo ajustado atendeu de forma satisfatória aos propósitos de se obter maior conhecimento acerca do mercado de soja, objeto de interesse deste estudo, apresentando resultados que podem contribuir para uma melhor compreensão do desempenho desse segmento.

Palavras-chave: Soja; Renda; Abate de Suínos e de Aves; VEC.

\section{ABSTRACT}

The objective of this paper is to analyze the main factors that influence the domestic soybean processing, based on monthly data from Jan/2005 to Dec/2015. For this purpose, a theoretical model is developed and adjusted, whose hypotheses are tested using the Vector Error Correction (VEC) Methodology. The information obtained by observing these factors contribute to the existing literature on the soybean market, which is generally focused on the analysis of the relation between prices. The results indicate that, among the variables considered in the model, income is the one that has the greatest influence on domestic soybean processing, with a positive and more than proportional effect, suggesting that demand for processed products grows when the purchasing power increases. Income also affects strongly and positively the number of hogs and poultry slaughtered, indicating that as purchasing power increases, meat consumption tends to rise. In general, the adjusted model satisfactorily served the purposes of gaining a greater knowledge about the soybean market, object of interest in this study, showing results that may contribute to a better understanding of the behavior of this segment.

Keywords: Soybean; Income; Slaughters of Hogs and Poultry; VEC.

JEL Code: E21; Q13; C32. 


\section{INTRODUÇÃO}

A soja, conforme ressalta Souza et al. (2010), é uma das principais fontes de proteína vegetal do mundo e tem sido amplamente utilizada como alimento, tanto pelos seres humanos quanto pelos animais. Porém, para que possa ser utilizada, é necessário que ela passe por um processo de industrialização, a partir do qual são gerados vários produtos, sendo os mais conhecidos o farelo e o óleo de soja.

De acordo com dados da Associação Brasileira das Indústrias de Óleos Vegetais (ABIOVE, 2016), do grão esmagado, cerca de 76\% são convertidos em farelo e $19 \%$ em óleo de soja. O farelo, principal produto gerado no processo de esmagamento da soja, é destinado especialmente ao suprimento da cadeia produtiva de carnes, por ser um dos insumos fundamentais na elaboração de ração animal, sobretudo para alimentação de suínos e aves, além de animais domésticos, peixes e bezerros, podendo ser considerado um substituto do leite nesse último caso (IMEA, 2015).

Goes et al. (2013) ressaltam que a alimentação está entre os principais gastos na produção animal, podendo chegar à proporção de $70 \%$ a $80 \%$ do custo total. Esses autores recomendam usar $20 \%$ a $30 \%$ de farelo de soja e até $80 \%$ de milho na composição da ração para animais monogástricos, como suínos e aves, e para animais ruminantes, o suficiente para atender às exigências nutricionais de proteínas.

Já o óleo de soja, além de ser usado na alimentação direta de seres humanos, é a principal matéria-prima na produção de biodiesel, correspondendo em média a $81 \%$ do volume desse bicombustível (ANP, 2016). Até 2010, a mistura obrigatória de biodiesel no óleo diesel era de 5\%, em 2014 foi ampliada para 7\% (Lei 13.033/2014), em 2018 subiu para 9\%, e em 2019 chegará a 10\% (Lei 13.263/2016), o que levará a uma demanda ainda maior de óleo de soja para a produção desse biocombustível. A adição do biodiesel ao diesel contribuiu para a redução das exportações brasileiras do óleo de soja, visto que o supracitado setor se tornou uma nova opção de mercado.

Machado (2010) destaca que o principal responsável pela demanda global de soja é o farelo, especialmente por causa do aumento do consumo mundial de carnes (bovina, suína e de frango). Um exemplo típico é o caso da China: o fato de ser líder na produção e no consumo mundial de carne suína e um dos principais produtores e consumidores mundiais de carnes bovina e de frango contribui para a crescente demanda de soja nesse país, tanto que é o maior importador da soja brasileira (pois sua produção de soja em grãos não é capaz de suprir sua grande demanda) e o maior consumidor mundial de farelo de soja, de acordo com dados do Departamento de Agricultura dos Estados Unidos (USDA, 2018).

O autor também ressalta que o aumento da demanda externa pelo grão e seus derivados e a evolução da soja no Brasil se deram de maneira concomitante, o que permitiu o rápido crescimento das exportações brasileiras. Na safra 2016/17, conforme dados do USDA (2018), entre os 114,1 milhões de toneladas de soja produzidas pelo Brasil, 63,1 milhões 
foram destinadas à exportação e 40,9 milhões ao processo de esmagamento. $\mathrm{O}$ Brasil tem mantido a liderança nas exportações e a segunda posição na produção mundial de soja, além de estar entre os quatro maiores esmagadores mundiais dessa oleaginosa.

China, Estados Unidos, Argentina e Brasil têm dominado o esmagamento do grão e a produção mundial de farelo de soja, evidenciando que esses países possuem complexos agroindustriais bem estruturados para o processamento da oleaginosa. Na safra 2016/17, o Brasil produziu cerca de 31,7 milhões de toneladas de farelo de soja, consumiu 16,9 milhões e exportou 13,8 milhões de toneladas. Quanto ao óleo de soja, no mesmo período, o país produziu cerca de 7,9 milhões de toneladas, consumiu 6,6 milhões e exportou 1,2 milhão de toneladas, tanto que o Brasil é o segundo maior exportador de farelo e óleo de soja e um dos quatro maiores produtores e consumidores mundiais desses dois produtos (USDA, 2018).

Assim, da soja produzida no Brasil, cerca de 36\% são destinados ao esmagamento do grão, sendo que o país consome grande parte dos derivados que produz, em torno de $53 \%$ do farelo e $84 \%$ do óleo de soja. O fato de o país ser um dos principais produtores e consumidores mundiais de carnes (bovina, suína e de frango) exige volumes crescentes de farelo de soja para produção de ração animal (USDA, 2018). Com relação ao óleo de soja, sua produção destina-se especialmente ao mercado interno para suprir a demanda da frota automotiva do país movida a diesel, por ser a principal matéria-prima na produção de biodiesel. Ressalta-se que o país é o segundo maior produtor e consumidor mundial desse bicombustível (BRASIL, 2016).

Existe uma gama de trabalhos nacionais e internacionais relacionados ao mercado de soja. Entre eles, vale citar os de Liu (2005), Brandão e Lima (2006), Tonin e Barczsz (2007), Sousa e Campos (2009), Silva e Machado (2009), Karali (2012), Le Clech (2013), Silva et al. (2013), Avalos (2014), Galvão et al. (2015), Bini et al. (2016), Alexakis et al. (2017) e Alves et al. (2018), que versam sobre aspectos referentes a preços. Já trabalhos como os de Filgueiras et al. (2007), Padrão et al. (2012), Pintor e Piacenti (2016) e Artuzo et al. (2018), abordam o setor produtivo de soja, enquanto os de Barros et al. (2002), Sampaio et al. (2004), Souza et al. (2007), Coronel et al. (2009), Feistel et al. (2015) e Souza et al. (2018), entre outros, focam suas análises nas exportações brasileiras de soja.

Todos esses estudos foram desenvolvidos com base em diversos tipos de modelagem, envolvendo métodos variados para análise de mercados, e auxiliarão no desenvolvimento do modelo proposto neste artigo, seja na abordagem metodológica seja na decisão sobre as variáveis a serem incluídas no modelo. No entanto, o que se observa é uma escassez de trabalhos voltados especificamente para o setor de esmagamento doméstico de soja, e os que o fazem, geralmente, voltam-se para a economia industrial, como o trabalho de Sediyama et al. (2013), e para a indústria da soja de uma forma mais ampla, como o de Figueiredo (2016).

Diante do exposto e considerando o fato de o país ser um dos principais esmagadores mundiais de soja, consumir grande parte dos derivados que 
produz e ser o segundo maior exportador de farelo e óleo de soja, um estudo com foco na análise desse segmento e nos fatores que causam maior impacto sobre o setor pode contribuir de forma considerável para melhor entender o comportamento do mercado de esmagamento de soja no Brasil.

Assim, este artigo tem como objetivo principal identificar e analisar os fatores que causam maior influência sobre o processamento de soja no Brasil, com base em dados de séries mensais do período de janeiro de 2005 a dezembro de 2015, por meio do desenvolvimento e ajuste de um modelo teórico. Espera-se que as informações sobre o mercado de soja obtidas no presente artigo possam complementar as de muitos outros trabalhos existentes na literatura, os quais versam, particularmente, sobre a questão da formação e da transmissão de preços. Adicionalmente, será construído um índice de preços dos derivados de soja para representar os preços dos principais produtos obtidos a partir do grão processado, do farelo e do óleo de soja, sendo também essa uma contribuição do presente artigo.

Além desta introdução, este artigo exibe o modelo teórico proposto, a metodologia, assim como a fonte e o tratamento dos dados utilizados para a realização das análises, expõe os principais resultados e discussões e encerra com as considerações finais.

\section{MODELO TEÓRICO PROPOSTO}

Para analisar os fatores que influenciam o processamento de soja no Brasil foram consideradas as seguintes variáveis no modelo teórico proposto:

$$
Q_{t}^{\text {proc }}=f\left(p a_{t}, \text { ipderiv }_{t}, \text { pex }_{t-1}^{\text {far }}, q_{t}^{\text {suínos }}, q_{t}^{\text {aves }}, y_{t}\right)
$$

em que $Q^{\text {proc }}$ representa a quantidade de soja processada no mercado interno, pa é o preço ao atacado do grão, ipderiv representa o índice de preços dos derivados de soja no mercado interno, pex far é o preço FOB de exportação do farelo de soja, expresso em reais, $q^{\text {suinos }}$ e $q^{\text {aves }}$ representam, respectivamente, as quantidades abatidas de suínos e de aves no mercado interno, e $y$ é a renda nacional.

A escolha dessas variáveis baseou-se nas características do mercado de soja e na literatura especializada. O índice de preços dos derivados de soja (ipderiv) foi construído para representar o farelo e o óleo de soja, principais produtos obtidos a partir do esmagamento da oleaginosa, como mencionado na introdução deste artigo. Tal índice foi construído com base na média ponderada dos preços ao atacado do farelo e do óleo de soja, tendo como pesos a participação de cada um desses produtos no processamento doméstico de soja em grãos. Essas participações foram obtidas dividindo-se a produção de cada bem (farelo e óleo) pela quantidade de soja processada. Em seguida, calculou-se a média dos valores encontrados em cada ano, ou seja, de 2005 a 2015, obtendo-se assim os seguintes valores: 0,76 para o farelo e 0,19 para o óleo de soja, considerando, então, $5 \%$ de perdas. 
Voltando à escolha das variáveis, o preço de exportação do farelo de soja foi incluído no modelo pelo fato de ser ele o principal produto industrializado da cadeia da soja exportado pelo Brasil. Na safra 2016/17, de acordo com dados do USDA (2018), o Brasil exportou cerca de $44 \%$ do farelo que produziu, enquanto essa relação para o óleo foi de apenas $15 \%$. Conforme visto anteriormente, grande parte do óleo de soja produzido no Brasil é utilizada na produção de biodiesel, principalmente para consumo interno, tanto que o país é o segundo maior produtor e consumidor mundial desse biocombustível (ANP, 2016; BRASIL, 2016).

De outro lado, a maior demanda mundial pelo farelo, em função do aumento do consumo de carnes, impulsionou as exportações desse derivado de soja. No período de 2016/17, segundo dados do USDA (2018), o consumo mundial de farelo foi de cerca de 222,3 milhões de toneladas, enquanto o de óleo de soja foi de 53,6 milhões de toneladas. Adicionalmente, as quantidades abatidas de suínos e de aves foram incluídas no modelo em função de o farelo de soja ser usado, sobretudo, na alimentação desses dois animais (IMEA, 2015).

Assim, a função (1), referente ao processamento doméstico de soja, constitui a base da matriz de relações contemporâneas do modelo proposto. Também foi considerada no modelo a influência da renda nacional nas quantidades abatidas de suínos e de aves no mercado interno. Espera-se que o preço da soja em grãos, assim como as quantidades abatidas de suínos e de aves no mercado interno, afete negativamente o processamento doméstico de soja. Já o índice de preços dos derivados de soja e a renda nacional, devem afetar positivamente a quantidade de soja processada no mercado interno. Além disso, a renda deve influenciar positivamente as quantidades abatidas de suínos e de aves, pois espera-se que um aumento na renda leve a um aumento no consumo de carnes.

\section{METODOLOGIA}

A abordagem econométrica usada para testar as hipóteses do modelo teórico proposto é a Análise de Autorregressão Vetorial (VAR), com identificação pelo procedimento de Bernanke, considerando as propriedades de integração e cointegração das séries temporais utilizadas. Bernanke (1986) propôs a identificação de um modelo VAR com base na matriz de relações contemporâneas, sendo possível estimar modelos sobreidentificados, com restrições além das necessárias. Para avaliar os efeitos de variações nos fatores que causam maior impacto sobre o processamento doméstico de soja, pretende-se utilizar instrumentais analíticos dessa metodologia, como função de resposta a impulsos e decomposição da variância dos erros de previsão.

A metodologia VAR é um método de modelos multivariados que foi inicialmente proposto por Sims (1980) e, segundo ressalta Bacchi (2007), tem sido muito utilizada em análises de assuntos macroeconômicos e em estudos relacionados ao agronegócio, devido à sua eficácia na estimação

Revista de Economia e Agronegócio - REA | V. 17 | N. 3 | 2019 | pág. 489 
de modelos que envolvem inter-relações complexas de variáveis e à grande variedade de resultados gerados, o que viabiliza uma maior riqueza na análise das inter-relações das variáveis de interesse.

Enders (2015) destaca que a metodologia VAR verifica se uma série temporal é afetada por valores presentes e passados de outra série, a fim de caracterizar a influência de cada variável sobre a outra no curto prazo. Assim, o objetivo desta análise é determinar as relações entre as variáveis do modelo e não as estimativas do parâmetro em si.

Para a aplicação da referida metodologia, é necessário que as séries sejam todas estacionárias. Para isso, o primeiro passo é determinar o número de raízes unitárias existentes na série, ou seja, sua ordem de integração. Entre os diversos procedimentos existentes para testar a presença de raiz unitária, optou-se por utilizar o desenvolvido por Elliott et al. (1996), conhecido como teste DF-GLS (Dickey Fuller Generalized Least Square), por ser mais robusto que o de Dickey-Fuller Aumentado (ADF), quando o processo gerador da série apresenta média e tendência não observáveis, além de se desenvolver satisfatoriamente em pequenas amostras, visto que o teste ADF foi desenvolvido para grandes amostras.

As modificações propostas por Elliott et al. (1996) aos testes de DickeyFuller fundamentam-se em dois aspectos: no fato de a remoção da média (constante) e da tendência de uma série utilizando mínimos Quadrados Ordinários (MQO) não ser eficiente e na necessidade de uma melhor aproximação do modelo especificado ao verdadeiro processo gerador de dados, por meio de uma seleção apropriada para a ordem de defasagem do modelo. Desse modo, os autores propuseram usar Mínimos Quadrados Generalizados (MQG) para remover a média e/ou a tendência da série $y_{t}$. Assim, a série filtrada é dada por:

$$
y_{t}^{d}=y_{t}-z_{t} \beta
$$

sendo que $y_{t}^{d}$ representa a série $y_{t}$ com média e/ou tendência removidas, dado que $z_{t}=(1, t)$ para remoção de média e tendência, e $z_{t}=1$ para remoção somente da média; e $\beta$ é o vetor de coeficientes da regressão, obtido da estimação de $\tilde{y}_{t}=\left[y_{1},(1-\bar{\rho} L) y_{2}, \ldots,(1-\bar{\rho} L) y_{T}\right]^{\prime}$ sobre $\tilde{z}_{t}=$ $\left[z_{1},(1-\bar{\rho} L) z_{2}, \ldots,(1-\bar{\rho} L) z_{T}\right]^{\prime}$, em que $L$ é o operador de defasagem, ou seja, $\mathrm{L} z_{t}=z_{t-1}$.

De posse da série filtrada, emprega-se o procedimento padrão ADF de teste de hipótese, apresentado em Dickey e Fuller (1979, 1981), para estimar a seguinte equação:

$$
\Delta y_{t}^{d}=\delta_{0} y_{t-1}^{d}+\sum_{i=2}^{p} \delta_{i} \Delta y_{t-i+1}^{d}+\zeta_{t}^{d}
$$

Na equação (3), estimada por $\mathrm{MQO}, y_{t}^{d}$ representa a série com média e/ou tendência removidas por MQG. O número de defasagens, $p$, é determinado pelo Critério Modificado de Akaike (MAIC), desenvolvido por $\mathrm{Ng}$ e Perron (2001), visto que os tradicionais critérios de informação, AIC e SC, tendem a selecionar baixos valores para as defasagens quando 
se tem raízes unitárias negativas (próximas a -1), levando a conclusões distorcidas.

Caso as séries não sejam estacionárias, mas sejam todas integradas de mesma ordem, o próximo passo é verificar a relação de cointegração entre elas, para então ajustar um modelo por meio do mecanismo de correção de erros, com as séries diferenciadas. A cointegração é útil para indicar se existe ou não uma relação de equilíbrio de longo prazo entre as variáveis.

Um dos testes bastante utilizados para verificar a existência de vetores de cointegração é o teste de cointegração de Johansen (1988), que utiliza estimadores de máxima verossimilhança e possibilita avaliar mais de duas variáveis explicativas simultaneamente, permitindo testar a presença de múltiplos vetores de cointegração, ao contrário do teste de Engle-Granger (1987), usado quando se tem apenas duas variáveis. Enders (2015) assinala que a metodologia de Johansen é uma generalização do teste de DickeyFuller num contexto multivariado. Para identificar o número de vetores de cointegração, utiliza-se a estatística traço e o teste de máximo autovalor.

Após determinar o número de vetores de cointegração, ajusta-se um Modelo de Autorregressão Vetorial com Correção de Erros (VEC), conciliando assim movimentos de curto prazo com equilíbrios de longo prazo. O modelo VEC pode ser descrito da seguinte forma:

$$
\Delta X_{t}=A_{0}+\Gamma_{1} \Delta X_{t-1}+\Gamma_{2} \Delta X_{t-2} \ldots+\Gamma_{p-1} \Delta X_{t-p+1}+\Pi X_{t-1}+\varepsilon_{t}
$$

em que: $\Delta X_{t}=X_{t}-X_{t-1}, \quad \Gamma_{i}=-\left(I-A_{1}-A_{2}-\cdots-A_{i}\right), \quad i=1, \ldots, p-1 \mathrm{e}$ $\Pi=\left(I-A_{1}-A_{2}-\cdots-A_{p}\right)$.

Após determinar o posto de $\Pi$, que corresponde ao número de vetores de cointegração, determinados por meio do teste de cointegração de Johansen, restringe-se essa matriz para construir o modelo de correção de erro. A matriz $\Pi$, apresentada em (4), pode ser representada por $\Pi=\alpha \beta^{\prime}$. Dessa forma, o termo de correção de erro é dado por:

$$
\Pi X_{t-1}=\alpha \beta^{\prime} X_{t-1}
$$

em que $a$ é a velocidade de ajustamento dos parâmetros da matriz no curto prazo, $\beta^{\prime}$ é uma matriz de coeficientes de cointegração de longo prazo e $\beta^{\prime} X_{t-1}$ representa até $(n-1)$ relações de cointegração no modelo multivariado, assegurando assim a convergência de $X_{t}$ para uma solução de equilíbrio de longo prazo.

Conforme enfatizado por Bacchi (2007), o uso da metodologia VAR permite analisar o mecanismo de transmissão de choques aleatórios sobre determinada variável com base na análise da reposta ao impulso, o que possibilita explicar alterações nas trajetórias das variáveis. De acordo com Enders (2015), a representação da função de resposta a impulso pode ser feita por meio de um vetor de médias móveis, em que as variáveis $x_{1 t}$ e $x_{2 t}$ são expressas em termos dos valores presentes e passados correspondentes aos erros $e_{1 t}$ e $e_{2 t}$. Desse modo, considerando um modelo bivariado, o modelo de média móvel é dado por: 


$$
X_{t}=\mu+\sum_{i=0}^{\infty} A_{1}^{i} e_{t-i}
$$

em que $\mu=\left[\begin{array}{ll}\bar{x}_{1} & \bar{x}_{2}\end{array}\right]$. Em termos de choques do modelo VAR estrutural, $\varepsilon_{1 t}$ e $\varepsilon_{2 t}$, tem-se:

$$
X_{t}=\mu+\sum_{i=0}^{\infty} \Phi_{i} \varepsilon_{t-i}
$$

em que $\phi_{i}=\left[\left(\frac{A_{1}^{i}}{1-\mathrm{b}_{12} \mathrm{~b}_{21}}\right)\right]\left[\begin{array}{cc}1 & -b_{12} \\ -b_{21} & 1\end{array}\right]$

A representação da média móvel é utilizada para examinar as interações entre as variáveis $x_{1 t}$ e $x_{2 t}$. Os coeficientes $\phi_{i}$ são usados para gerar os efeitos dos choques em $\varepsilon_{1 t}$ e $\varepsilon_{2 t}$, sendo que os elementos $\phi_{j k}(i)$, denominados multiplicadores de impacto, medem o impacto da mudança de uma unidade de $\varepsilon_{1 t}$ e $\varepsilon_{2 t}$ sobre $x_{1 t}$ e $x_{2 t}$, respectivamente.

Bacchi (2007) ressalta que a metodologia VAR também permite decompor a variância dos erros de previsão $k$ períodos à frente em percentuais a serem atribuídos a cada variável do sistema, bem como possibilita avaliar o poder explicativo de cada variável sobre as demais. Trata-se de uma forma alternativa de medir os efeitos dos choques sobre as variáveis do modelo. Retomando a equação (7), $n$ períodos à frente, para se obter os erros de previsão em termos de choques do modelo VAR estrutural, temse:

$$
X_{t+n}=\mu+\sum_{i=0}^{\infty} \Phi_{i} \varepsilon_{t+n-i}
$$

Assim, o erro de previsão, $n$ períodos à frente, será:

$$
\sum_{i=0}^{n-1} \Phi_{i} \varepsilon_{t+n-i}=X_{t+n}-E X_{t+n}
$$

Considerando novamente, como exemplo, um modelo VAR bivariado, a variância do erro de previsão, $n$ períodos à frente, pode ser denotada por $\sigma_{x_{1}}(n)^{2}$ :

$$
\begin{gathered}
\sigma_{x_{1}}(n)^{2}=\sigma_{x_{1}}^{2}\left[\phi_{11}(0)^{2}+\phi_{11}(1)^{2}+\cdots+\phi_{11}(n-1)^{2}\right]+ \\
\sigma_{x_{2}}^{2}\left[\phi_{12}(0)^{2}+\phi_{12}(1)^{2}+\cdots+\phi_{12}(n-1)^{2}\right]
\end{gathered}
$$

Por fim, a decomposição da variância do erro de previsão pode ser representada por:

$$
\frac{\sigma_{x_{1}}^{2}\left[\phi_{11}(0)^{2}+\phi_{11}(1)^{2}+\cdots+\phi_{11}(n-1)^{2}\right]}{\sigma_{x_{1}}(n)^{2}}
$$




$$
\frac{\sigma_{x_{2}}^{2}\left[\phi_{12}(0)^{2}+\phi_{12}(1)^{2}+\cdots+\phi_{12}(n-1)^{2}\right]}{\sigma_{x_{1}}(n)^{2}}
$$

Essa decomposição indica a proporção do movimento de uma variável em função de um choque nela própria contra os choques em outras variáveis do sistema. Bacchi (2007) enfatiza que a sequência $\left\{x_{1 t}\right\}$ será exógena se os choques $\varepsilon_{2 t}$ não explicarem nenhuma parcela da variância do erro de previsão de $\left\{x_{1 t}\right\}$ ao longo de todo o período previsto. Entretanto, se aqueles choques conseguirem explicar toda a variância do erro de previsão de $\left\{x_{1 t}\right\}$, essa sequência será endógena. A autora observa que em geral, no curto prazo, uma variável consegue explicar grande parte da variância do seu próprio erro de previsão, no entanto, essa proporção diminui num horizonte de previsão maior.

\section{FONTE E TRATAMENTO DOS DADOS}

Para o desenvolvimento das análises, foram usados dados mensais de janeiro de 2005 a dezembro de 2015, todos transformados em logaritmo, de modo que as relações entre as variáveis pudessem ser interpretadas como elasticidades. Além disso, todas as variáveis foram deflacionadas pelo IGP-DI (base de dezembro de 2015).

A fonte e as unidades de medida das variáveis consideradas no modelo proposto são apresentadas na Tabela 1.

Tabela 1. Detalhamento das séries empregadas no modelo de processamento de soja no Brasil

\begin{tabular}{|c|c|c|c|}
\hline Variável & Descrição da variável & Unidade & Fonte \\
\hline pa & Preço físico da soja em grãos - Paraná & $\mathrm{R} \$ / \mathrm{sc} 60 \mathrm{~kg}$ & CEPEA/ESALQ \\
\hline pafar & $\begin{array}{c}\text { Preço físico do farelo de soja - Ponta } \\
\text { Grossa (RS) }\end{array}$ & $\mathrm{R} \$ / \mathrm{t}$ & CEPEA/ESALQ \\
\hline paoleo & Preço físico do óleo de soja - São Paulo & $\mathrm{R} \$ / \mathrm{t}$ & CEPEA/ESALQ \\
\hline pexfar & Preço FOB do farelo de soja - Paranaguá & $\mathrm{US} \$ / \mathrm{t}$ & $\begin{array}{l}\text { CEPEA/BM\&F } \\
\text { BOVESPA }\end{array}$ \\
\hline prodfar & Produção anual de farelo de soja & mil t & CONAB \\
\hline prodoleo & Produção anual de óleo de soja & mil $\mathrm{t}$ & CONAB \\
\hline qproc & $\begin{array}{l}\text { Quantidade mensal de soja em grãos } \\
\text { processada }\end{array}$ & mil t & ABIOVE \\
\hline qsuinos & Quantidade mensal abatida de suínos & cabeças & IBGE \\
\hline qaves & Quantidade mensal abatida de aves & cabeças & IBGE \\
\hline $\mathrm{y}$ & Renda nacional (PIB mensal) & $\mathrm{R} \$$ (milhões) & IPEA \\
\hline câmbio & $\begin{array}{c}\text { Taxa de câmbio comercial de venda } \\
\text { (média } \mathrm{R} \$ \text { ) }\end{array}$ & $\mathrm{R} \$ / \mathrm{US} \$$ & IPEA \\
\hline IGP-DI & $\begin{array}{l}\text { Índice geral de preços-disponibilidade } \\
\text { interna (Base do } \mathrm{n}^{\circ} \text { índice } 01 / 08 / 1994 \text { ) }\end{array}$ & - & FGV \\
\hline
\end{tabular}

Fonte: Elaborado pelos autores. 


\section{RESULTADOS E DISCUSSÃO}

Os resultados apresentados na Tabela 2 mostram que, para todas as séries analisadas, não há evidências para rejeitar a hipótese nula de presença de uma raiz unitária, seja no caso de inclusão apenas de constante (Modelo B), seja no caso de inclusão de constante e tendência (Modelo A). Assim, todas as variáveis do modelo são integradas de ordem um, I (1). A Tabela 3, por sua vez, refere-se aos resultados dos testes de cointegração do modelo proposto. Pode-se concluir, ao nível de 5\% de significância, que existem três vetores de cointegração.

Tabela 2. Resultados dos testes de raiz unitária (DF-GLS) para o modelo de processamento de soja no Brasil, janeiro de 2005 a dezembro de 2015

\begin{tabular}{lcccc}
\hline \multirow{2}{*}{ Variável } & \multicolumn{2}{c}{ Modelo A } & \multicolumn{2}{c}{ Modelo B } \\
\cline { 2 - 5 } & Defasagens & Estatística & Defasagens & Estatística \\
& $\mathrm{p}$ & DF-GLS & $\mathrm{p}$ & DF-GLS \\
\hline Quantidade de soja processada & 12 & $-1,818$ & 11 & 2,112 \\
Preço ao atacado do grão & 8 & $-2,077$ & 0 & $-1,573$ \\
Índice de preços dos derivados de soja & 8 & $-2,098$ & 0 & $-1,888$ \\
Preço de exportação do farelo & 2 & $-2,595$ & 0 & $-1,750$ \\
Quantidade abatida de suínos & 12 & $-0,843$ & 7 & 1,814 \\
Quantidade abatida de aves & 11 & $-1,058$ & 12 & 1,490 \\
Renda nacional & 12 & $-0,746$ & 5 & 0,413 \\
\hline
\end{tabular}

Fonte: Resultados da pesquisa.

Nota: Modelo A: versão com constante e tendência - valores críticos em Elliott, Rothenberg e Stock (1996) $(10 \%=-2,64,5 \%=-2,93,1 \%=-3,46)$; Modelo B: na versão apenas com constante - valores críticos em Dickey e Fuller $(1979 ; 1981)(10 \%=-1,62,5 \%=$ $-1,95$ e $1 \%=-2,58)$.

Tabela 3. Resultados dos testes de cointegração de Johansen para o modelo de processamento de soja no Brasil

\begin{tabular}{ccccc}
\hline $\begin{array}{c}\text { Hipótese } \\
\text { Nula }\end{array}$ & $\begin{array}{c}\text { Hipótese } \\
\text { Alternativa }\end{array}$ & $\begin{array}{c}\text { Estatística } \\
\text { Traço }\end{array}$ & $\begin{array}{c}\text { Valores } \\
\text { Críticos }(5 \%)\end{array}$ & P-Valor \\
\hline $\mathrm{r}=0$ & $\mathrm{r}>0$ & 235,457 & 125,417 & 0,000 \\
$\mathrm{r} \leq 1$ & $\mathrm{r}>1$ & 135,134 & 95,514 & 0,000 \\
$\mathrm{r} \leq 2$ & $\mathrm{r}>2$ & 85,596 & 69,611 & 0,001 \\
$\mathrm{r} \leq \mathbf{3}$ & $\mathrm{r}>3$ & $\mathbf{4 1 , 1 8 9}$ & $\mathbf{4 7 , 7 0 7}$ & $\mathbf{0 , 1 8 4}$ \\
$\mathrm{r} \leq 4$ & $\mathrm{r}>4$ & 19,018 & 29,804 & 0,502 \\
$\mathrm{r} \leq 5$ & $\mathrm{r}>5$ & 8,807 & 15,408 & 0,391 \\
$\mathrm{r} \leq 6$ & $\mathrm{r}>6$ & 3,169 & 3,841 & 0,075 \\
\hline
\end{tabular}

Fonte: Resultados da pesquisa.

Assim, o modelo proposto para identificar os determinantes que causam maiores impactos sobre o processamento doméstico de soja em grãos foi construído com uma defasagem e as seguintes variáveis: quantidade de soja processada, preço ao atacado de soja em grãos, índice de preços dos derivados de soja, preço FOB de exportação do farelo de soja, quantidade abatida de suínos, quantidade abatida de aves e renda nacional; todas 
transformadas em logaritmo e na primeira diferença. Além da constante e dos três vetores de correção de erros, foram incluídas no modelo variáveis binárias para controlar o efeito sazonal.

As influências contemporâneas de cada variável considerada no modelo são apresentadas na Tabela 4 . Os resultados indicam que os sinais de todos os coeficientes estimados estão conforme o esperado. A maioria das relações é estatisticamente significativa ao nível de 10\% de probabilidade, com exceção das relações do preço do grão ao atacado, do índice de preços dos derivados de soja e do abate de aves sobre a quantidade de soja processada ${ }^{1}$. Das variáveis consideradas no modelo, a renda é a que exerce maior influência contemporânea sobre a quantidade de soja processada no mercado interno, cuja transmissão é mais do que proporcional. Nota-se ainda a influência da renda transmitida quase integralmente para as quantidades abatidas de suínos e de aves.

Tabela 4. Coeficientes estimados pela matriz de relações contemporâneas do modelo de processamento de soja no Brasil, por meio de um VEC, dados mensais

\begin{tabular}{|c|c|c|c|c|c|}
\hline \multicolumn{2}{|c|}{ Relações Contemporâneas } & \multirow{2}{*}{$\begin{array}{c}\text { Coeficientes } \\
\text { Estimados }\end{array}$} & \multirow{2}{*}{$\begin{array}{l}\text { Desvio } \\
\text { Padrão }\end{array}$} & \multirow{2}{*}{$\begin{array}{c}\text { Estatística } \\
\mathrm{t}\end{array}$} & \multirow{2}{*}{ Significância } \\
\hline$\overline{\mathrm{De}}$ & Sobre & & & & \\
\hline Preço do grão ao atacado & Soja processada & $-0,147$ & 0,329 & $-0,446$ & 0,655 \\
\hline $\begin{array}{l}\text { Índice de preços dos } \\
\text { derivados de soja }\end{array}$ & Soja processada & 0,127 & 0,356 & 0,357 & 0,721 \\
\hline Abate de suínos & Soja processada & $-0,548$ & 0,288 & $-1,902$ & 0,057 \\
\hline Abate de aves & Soja processada & $-0,040$ & 0,247 & $-0,160$ & 0,873 \\
\hline Renda & Soja processada & 1,712 & 0,324 & 5,291 & 0,000 \\
\hline Renda & Abate de suínos & 0,878 & 0,124 & 7,111 & 0,000 \\
\hline Renda & Abate de aves & 0,832 & 0,143 & 5,835 & 0,000 \\
\hline
\end{tabular}

Fonte: Resultados da pesquisa.

A Figura 1 apresenta as respostas acumuladas da quantidade de soja processada a choques nela mesma e nas demais variáveis consideradas no modelo, doze meses à frente. Como se observa, um choque exógeno de $1 \%$ na renda causa impacto acumulado positivo, com transmissão mais do que proporcional para a quantidade de soja processada, confirmando o resultado obtido no coeficiente estimado pela matriz de relações contemporâneas. Outra variável que impacta a quantidade de soja processada é a quantidade abatida de suínos, embora nesse caso o efeito acumulado seja negativo e menos do que proporcional, assim como os efeitos acumulados de choques não esperados no preço ao atacado da soja em grãos e na quantidade abatida de aves.

1 Também foi ajustado um modelo para o processamento doméstico de soja sem considerar contemporaneamente o abate de suínos e de aves sobre a quantidade de soja processada. No entanto, o modelo alternativo não apresentou melhores resultados. 
Já um choque não antecipado de $1 \%$ no índice de preços dos derivados de soja, afeta positivamente a quantidade de soja processada, como era de se esperar, embora esses efeitos acumulados sejam de menor magnitude, se comparados aos efeitos da renda. Esse resultado corrobora o enfatizado por Barros (2012), que observa que um aumento relativo no preço dos derivados tende a aumentar sua oferta na medida em que a produção se expande.

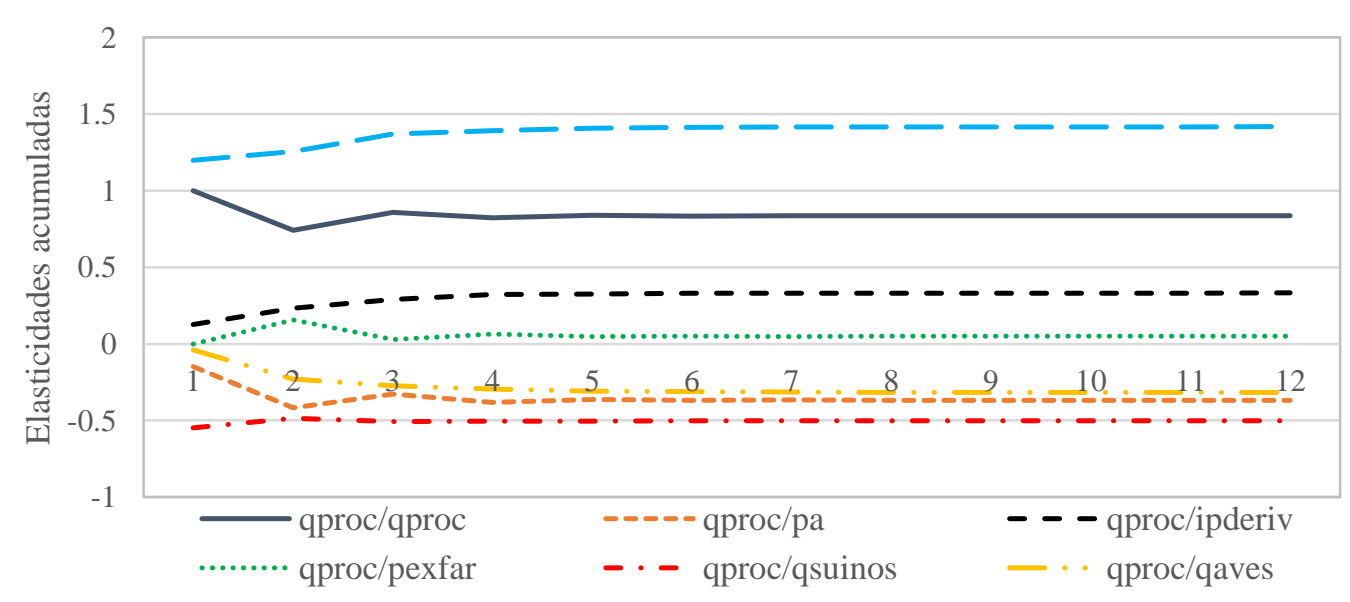

Figura 1. Respostas acumuladas da quantidade de soja processada (qproc) a choques não antecipados na própria variável (qproc), no preço ao atacado do grão (pa), no índice de preços dos derivados de soja (ipderiv), no preço de exportação do farelo de soja (pexfar), nas quantidades abatidas de suínos (qsuinos) e de aves (qaves) e na renda nacional (y)

Fonte: Resultados da pesquisa.

Também é possível observar, na Figura 1, que os efeitos dos choques de todas as variáveis consideradas na análise sobre a quantidade de soja processada se estabilizam a partir do terceiro mês. Como os impactos são acumulados, pode-se concluir que os efeitos desses choques são temporários, visto que desaparecem a partir do terceiro mês.

Na Figura 2, pode-se verificar que um choque exógeno de $1 \%$ no preço ao atacado do grão afeta de forma positiva e com maior intensidade o preço de exportação do farelo de soja e o índice de preços dos derivados de soja, cujas magnitudes chegam a $0,5 \%$ e $0,4 \%$, respectivamente, no segundo período após o choque. Como visto anteriormente, o efeito acumulado de um choque não antecipado de $1 \%$ no preço doméstico do grão sobre a quantidade de soja processada é negativo. Esses resultados são pertinentes com o exposto por Barros (2012), o qual ressalta que um aumento no preço da matéria-prima tende a reduzir a oferta de produtos processados, elevando assim os seus preços.

Na Figura 3, observa-se que um impulso de 1\% no índice de preços dos derivados de soja causa um impacto acumulado positivo sobre a renda, ao passo que o efeito acumulado desse choque sobre as variáveis referentes à 
quantidade abatida de aves, ao preço de exportação do farelo de soja e ao preço do grão no mercado interno é negativo.
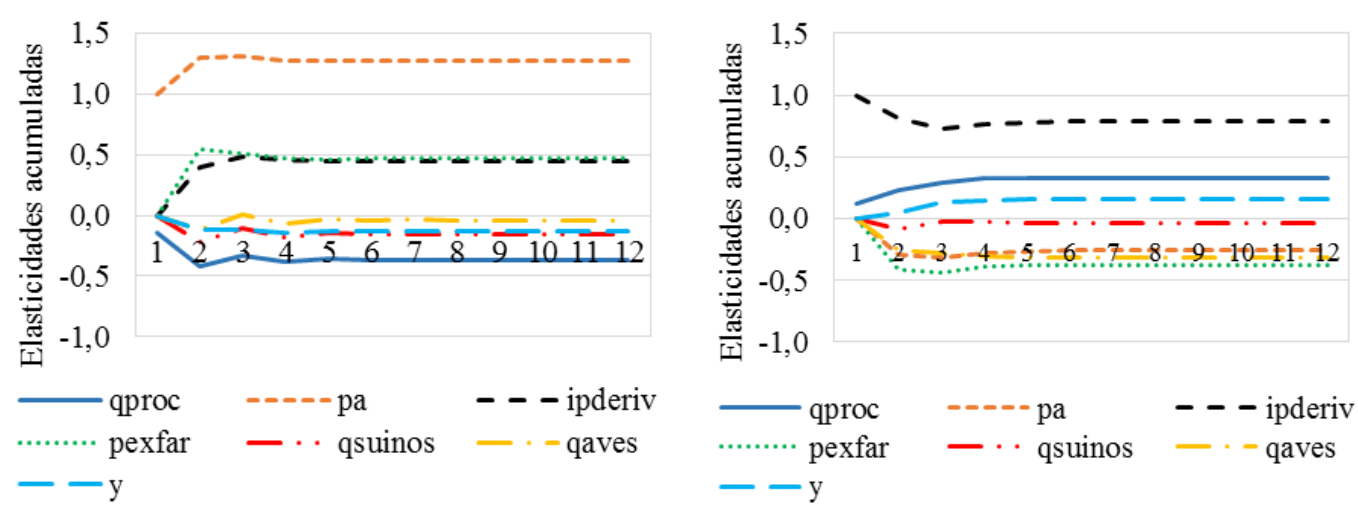

Figura 2. Respostas acumuladas das Figura 3. Respostas acumuladas das variáveis do modelo a um choque variáveis do modelo a um choque exógeno no preço ao atacado do exógeno no índice de preços dos grão (pa)

Fonte: Resultados da pesquisa. derivados de soja (ipderiv)

Fonte: Resultados da pesquisa.

Como se observa na Figura 4, a única variável que responde de forma negativa a um choque não antecipado de $1 \%$ no preço de exportação do farelo de soja é a renda. Cabe salientar que o impacto desse choque sobre todas as variáveis analisadas também é de pequena magnitude, sendo que o maior deles, sobre a quantidade abatida de aves, não ultrapassa $0,3 \%$ no acumulado.

Parece haver uma certa desproporcionalidade de transmissão entre o preço ao atacado do grão, o preço de exportação do farelo e o índice de preços dos derivados de soja. Enquanto o preço ao atacado do grão mostra certa relevância para explicar variações no preço dos derivados, tanto no mercado doméstico (ipderiv) quanto no preço de exportação (pexfar), o efeito inverso é menos acentuado, indicando que o preço dos derivados, ipderiv e pexfar, não têm tanta significância sobre o preço da matériaprima. Tal comportamento deve se justificar pelo tamanho de cada mercado, uma vez que o volume de soja em grãos é muito maior do que o processamento e, portanto, menos sensível.

Quanto à Figura 5, observa-se que um aumento de 1\% na quantidade abatida de suínos causa efeitos acumulados positivos sobre os três preços considerados no modelo, ou seja, preço de exportação do farelo de soja, preço ao atacado da soja em grãos e índice de preços dos derivados de soja. 

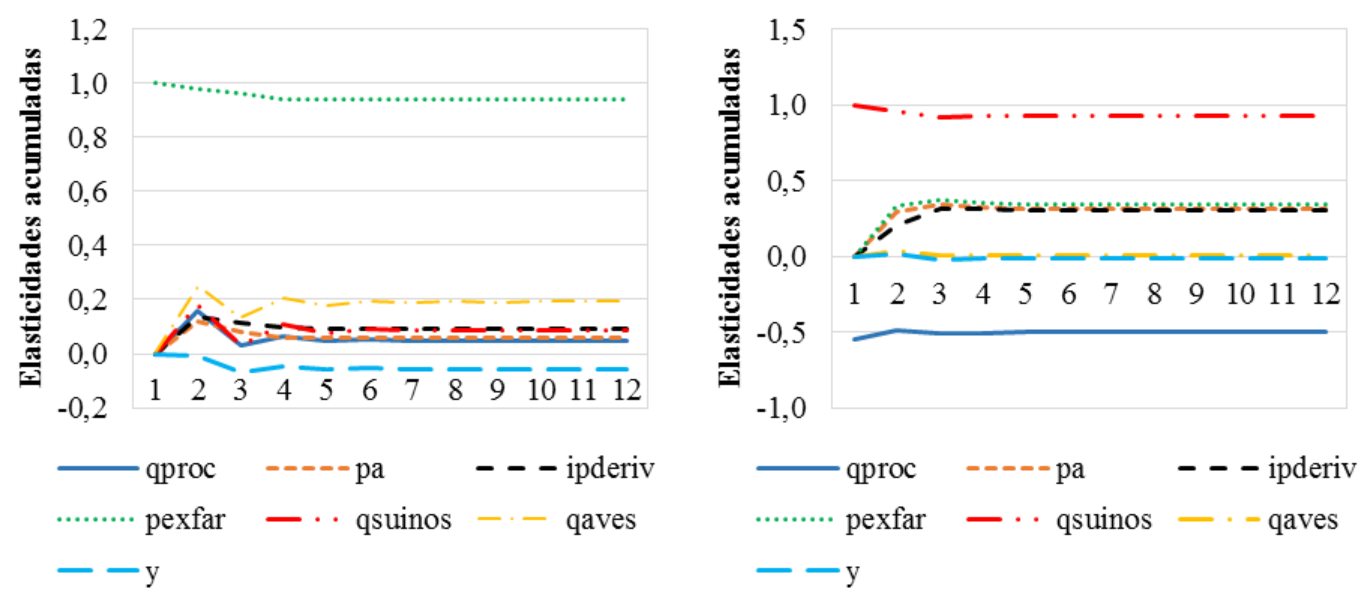

Figura 4. Respostas acumuladas das Figura 5. Respostas acumuladas das variáveis do modelo a um choque variáveis do modelo a um choque exógeno no preço de exportação do exógeno na quantidade abatida de farelo de soja (pexfar)

Fonte: Resultados da pesquisa. suínos (qsuinos)

Fonte: Resultados da pesquisa.

Já um choque exógeno de $1 \%$ na quantidade abatida de aves causa efeitos acumulados negativos sobre todas as variáveis, exceto sobre ela mesma, como pode ser constatado na Figura 6. As variáveis mais influenciadas por esse choque são os três preços considerados no modelo e a quantidade de soja processada, embora esses efeitos não ultrapassem $-0,5 \%$ no acumulado. No curto prazo, e para choques não antecipados, esses resultados são pertinentes, visto que quando o abate de aves aumenta, a demanda por farelo tende a diminuir no primeiro momento, pois a quantidade de animais para serem alimentados diminui, reduzindo a demanda por esmagamento de soja e, consequentemente, os preços dos seus derivados. Além disso, como a demanda por esmagamento de soja diminui, a oferta de grãos tende a aumentar, com consequente queda do seu preço no mercado interno.

Dois pontos devem ser considerados ao analisar os resultados das relações entre as quantidades abatidas de suínos e de aves e a quantidade de soja processada. O primeiro refere-se aos sinais dos coeficientes obtidos na matriz de relações contemporâneas para essas duas relações. Imediatamente após o abate de suínos e de aves, faz sentido a quantidade de soja processada diminuir, pois a demanda por ração e consequentemente por farelo, no curto prazo, tenderá a reduzir, visto que serão menos animais para serem alimentados, como mencionado anteriormente. Em segundo lugar, deve-se atentar ao fato de os dados dessas variáveis serem mensais. Possivelmente, se eles fossem anuais, os sinais dos coeficientes das referidas relações seriam positivos, visto que, quando o abate de suínos e de aves aumenta, há necessidade de se produzir (criar e engordar) mais animais, o que, por sua vez, leva a um aumento no consumo de ração até que eles fiquem prontos para o abate, 
aumentando assim a demanda por farelo e, consequentemente, por esmagamento de soja.

Já um choque não antecipado de 1\% na renda nacional (Figura 7), causa impactos positivos sobre as quantidades abatidas de suínos e de aves, com transmissão de impacto quase integral no primeiro período, cerca de $0,9 \%$ e $0,8 \%$, respectivamente; verifica-se, nesse caso, a elasticidade rendaconsumo de proteína tão preconizada na teoria econômica. Além disso, todas as variáveis referentes a preço respondem positivamente a choques não antecipados na renda, no entanto, esses efeitos são de pequena magnitude, não ultrapassando $0,3 \%$ no acumulado, sugerindo que à medida que a renda aumenta, a demanda por produtos processados também aumenta, elevando assim os seus preços.
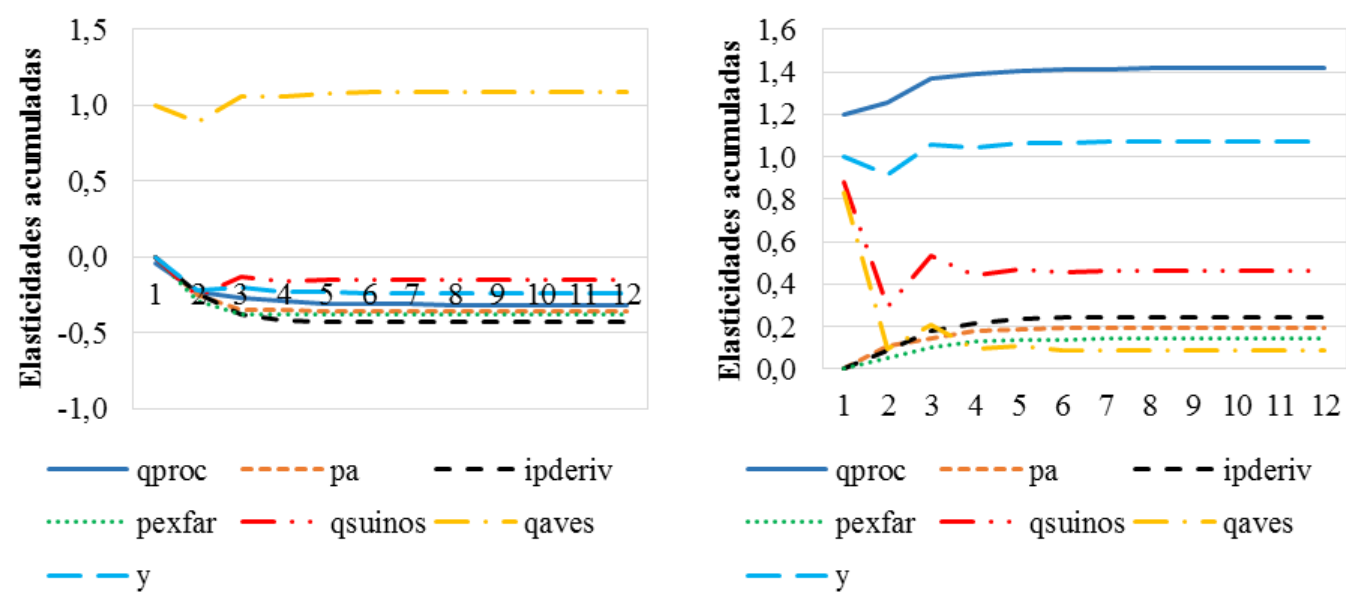

Figura 6. Respostas acumuladas das Figura 7. Respostas acumuladas das variáveis do modelo a um choque variáveis do modelo a um choque não antecipado na quantidade não antecipado na renda $(y)$ abatida de aves (qaves)

Fonte: Resultados da pesquisa.

Fonte: Resultados da pesquisa.

A seguir, são apresentadas, nas Tabelas 5, 6 e 7, as respectivas variâncias dos erros de previsão do processamento doméstico de soja e das quantidades abatidas de suínos e de aves, decompostas doze meses à frente. 
Tabela 5. Decomposição da variância do erro de previsão do processamento doméstico de soja

\begin{tabular}{ccccccccc}
\hline Período & Desvio Padrão & qproc & pa & ipderiv & pexfar & qsuinos & qaves & y \\
\hline 1 & 0,086 & $\mathbf{8 1 , 3 3 0}$ & 0,573 & 0,365 & 0,000 & 5,028 & 0,036 & $\mathbf{1 2 , 6 6 8}$ \\
2 & 0,090 & $\mathbf{7 9 , 1 0 2}$ & 2,288 & 0,560 & 1,041 & 4,648 & 0,780 & $\mathbf{1 1 , 5 8 2}$ \\
3 & 0,091 & $\mathbf{7 8 , 4 4 2}$ & 2,428 & 0,617 & 1,705 & 4,559 & 0,804 & $\mathbf{1 1 , 4 4 6}$ \\
4 & 0,091 & $\mathbf{7 8 , 3 3 6}$ & 2,492 & 0,635 & 1,758 & 4,547 & 0,811 & $\mathbf{1 1 , 4 2 1}$ \\
5 & 0,091 & $\mathbf{7 8 , 3 1 7}$ & 2,501 & 0,635 & 1,770 & 4,545 & 0,814 & $\mathbf{1 1 , 4 1 8}$ \\
6 & 0,091 & $\mathbf{7 8 , 3 1 5}$ & 2,502 & 0,635 & 1,771 & 4,545 & 0,814 & $\mathbf{1 1 , 4 1 7}$ \\
7 & 0,091 & $\mathbf{7 8 , 3 1 4}$ & 2,503 & 0,635 & 1,771 & 4,545 & 0,815 & $\mathbf{1 1 , 4 1 7}$ \\
8 & 0,091 & $\mathbf{7 8 , 3 1 4}$ & 2,503 & 0,635 & 1,771 & 4,545 & 0,815 & $\mathbf{1 1 , 4 1 7}$ \\
9 & 0,091 & $\mathbf{7 8 , 3 1 4}$ & 2,503 & 0,635 & 1,771 & 4,545 & 0,815 & $\mathbf{1 1 , 4 1 7}$ \\
10 & 0,091 & $\mathbf{7 8 , 3 1 4}$ & 2,503 & 0,635 & 1,771 & 4,545 & 0,815 & $\mathbf{1 1 , 4 1 7}$ \\
11 & 0,091 & $\mathbf{7 8 , 3 1 4}$ & 2,503 & 0,635 & 1,771 & 4,545 & 0,815 & $\mathbf{1 1 , 4 1 7}$ \\
12 & 0,091 & $\mathbf{7 8 , 3 1 4}$ & 2,503 & 0,635 & 1,771 & 4,545 & 0,815 & $\mathbf{1 1 , 4 1 7}$ \\
\hline
\end{tabular}

Fonte: Resultados da pesquisa.

Tabela 6. Decomposição da variância do erro de previsão da quantidade abatida de suínos

\begin{tabular}{ccccccccc}
\hline Período & Desvio Padrão & qproc & pa & ipderiv & pexfar & qsuinos & qaves & $\mathbf{y}$ \\
\hline 1 & 0,042 & 0,000 & 0,000 & 0,000 & 0,000 & $\mathbf{7 1 , 0 3 6}$ & 0,000 & $\mathbf{2 8 , 9 6 4}$ \\
2 & 0,049 & 5,379 & 3,839 & 0,505 & 4,608 & $\mathbf{5 0 , 9 9 9}$ & 4,610 & $\mathbf{3 0 , 0 6 1}$ \\
3 & 0,052 & 7,969 & 4,308 & 0,723 & 7,064 & $\mathbf{4 6 , 1 4 1}$ & 5,210 & $\mathbf{2 8 , 5 8 5}$ \\
4 & 0,052 & 8,646 & 4,601 & 0,711 & 7,711 & $\mathbf{4 5 , 0 5 3}$ & 5,162 & $\mathbf{2 8 , 1 1 7}$ \\
5 & 0,052 & 8,794 & 4,701 & 0,707 & 7,831 & $\mathbf{4 4 , 8 2 4}$ & 5,145 & $\mathbf{2 7 , 9 9 8}$ \\
6 & 0,053 & 8,827 & 4,718 & 0,707 & 7,855 & $\mathbf{4 4 , 7 8 0}$ & 5,141 & $\mathbf{2 7 , 9 7 3}$ \\
7 & 0,053 & 8,833 & 4,721 & 0,706 & 7,860 & $\mathbf{4 4 , 7 7 2}$ & 5,140 & $\mathbf{2 7 , 9 6 8}$ \\
8 & 0,053 & 8,834 & 4,721 & 0,706 & 7,860 & $\mathbf{4 4 , 7 7 0}$ & 5,140 & $\mathbf{2 7 , 9 6 8}$ \\
9 & 0,053 & 8,834 & 4,722 & 0,706 & 7,860 & $\mathbf{4 4 , 7 7 0}$ & 5,140 & $\mathbf{2 7 , 9 6 7}$ \\
10 & 0,053 & 8,834 & 4,722 & 0,706 & 7,860 & $\mathbf{4 4 , 7 7 0}$ & 5,140 & $\mathbf{2 7 , 9 6 7}$ \\
11 & 0,053 & 8,834 & 4,722 & 0,706 & 7,860 & $\mathbf{4 4 , 7 7 0}$ & 5,140 & $\mathbf{2 7 , 9 6 7}$ \\
12 & 0,053 & 8,834 & 4,722 & 0,706 & 7,860 & $\mathbf{4 4 , 7 7 0}$ & 5,140 & $\mathbf{2 7 , 9 6 7}$ \\
\hline
\end{tabular}

Fonte: Resultados da pesquisa.

Tabela 7. Decomposição da variância do erro de previsão da quantidade abatida de aves

\begin{tabular}{ccccccccc}
\hline Período & Desvio Padrão & qproc & pa & ipderiv & pexfar & qsuinos & qaves & y \\
\hline 1 & 0,046 & 0,000 & 0,000 & 0,000 & 0,000 & 0,000 & $\mathbf{7 8 , 9 0 6}$ & $\mathbf{2 1 , 0 9 4}$ \\
2 & 0,055 & 5,623 & 0,838 & 3,559 & 6,951 & 0,049 & $\mathbf{5 6 , 3 4 2}$ & $\mathbf{2 6 , 6 3 8}$ \\
3 & 0,057 & 6,806 & 1,652 & 3,387 & 7,975 & 0,072 & $\mathbf{5 4 , 6 5 4}$ & $\mathbf{2 5 , 4 5 6}$ \\
4 & 0,057 & 7,289 & 1,894 & 3,365 & 8,409 & 0,071 & $\mathbf{5 3 , 7 0 1}$ & $\mathbf{2 5 , 2 7 0}$ \\
5 & 0,057 & 7,373 & 1,958 & 3,360 & 8,464 & 0,071 & $\mathbf{5 3 , 5 7 6}$ & $\mathbf{2 5 , 1 9 9}$ \\
6 & 0,057 & 7,396 & 1,969 & 3,358 & 8,482 & 0,071 & $\mathbf{5 3 , 5 3 7}$ & $\mathbf{2 5 , 1 8 7}$ \\
7 & 0,057 & 7,399 & 1,971 & 3,358 & 8,484 & 0,071 & $\mathbf{5 3 , 5 3 3}$ & $\mathbf{2 5 , 1 8 4}$ \\
8 & 0,057 & 7,400 & 1,972 & 3,358 & 8,484 & 0,071 & $\mathbf{5 3 , 5 3 1}$ & $\mathbf{2 5 , 1 8 4}$ \\
9 & 0,057 & 7,400 & 1,972 & 3,358 & 8,484 & 0,071 & $\mathbf{5 3 , 5 3 1}$ & $\mathbf{2 5 , 1 8 3}$ \\
10 & 0,057 & 7,400 & 1,972 & 3,358 & 8,484 & 0,071 & $\mathbf{5 3 , 5 3 1}$ & $\mathbf{2 5 , 1 8 3}$ \\
11 & 0,057 & 7,400 & 1,972 & 3,358 & 8,484 & 0,071 & $\mathbf{5 3 , 5 3 1}$ & $\mathbf{2 5 , 1 8 3}$ \\
12 & 0,057 & 7,400 & 1,972 & 3,358 & 8,484 & 0,071 & $\mathbf{5 3 , 5 3 1}$ & $\mathbf{2 5 , 1 8 3}$ \\
\hline
\end{tabular}

Fonte: Resultados da pesquisa. 
Observa-se, na Tabela 5, que além da própria variável que explica, em média, $79 \%$ de suas variações, ao longo do tempo, a renda é o fator que mais contribui para variações no comportamento da quantidade de soja processada, seguida pelo abate de suínos, corroborando os resultados encontrados nos coeficientes estimados pela matriz de relações contemporâneas e nas análises das funções de respostas a impulsos. Juntas, essas duas variáveis conseguem explicar cerca de $16 \%$ das variações no processamento de soja, no final dos doze meses.

Quanto às Tabelas 6 e 7, observa-se que variações nas quantidades abatidas de suínos e de aves também são fortemente explicadas pela renda. No primeiro caso, a renda explica cerca de $28 \%$, e no segundo, cerca de $25 \%$, no final de doze meses. Esses resultados ratificam os encontrados nas análises das funções de respostas a impulsos, visto que, em geral, à medida que a renda aumenta, o consumo de carnes tende a aumentar, especialmente de carnes mais caras, como a suína. Embora menos relevantes, se comparados à renda, outros fatores que influenciam variações nos abates de suínos e de aves são a quantidade de soja processada e o preço de exportação do farelo.

\section{CONSIDERAÇÕES FINAIS}

Este artigo, que teve como objetivo identificar e analisar os fatores que causam maior influência sobre o processamento de soja no Brasil, atendeu de forma satisfatória aos propósitos de se obter um maior conhecimento acerca desse setor. As informações relacionadas ao mercado de soja obtidas no presente artigo complementam as de muitos outros trabalhos existentes na literatura, os quais versam, particularmente, sobre os aspectos referentes a preços. Não se tem conhecimento de nenhum trabalho na literatura que apresente a abordagem tratada aqui para o setor de processamento de soja, de forma que este artigo vem preencher essa lacuna. Outra contribuição do presente estudo é a construção de um índice de preços dos derivados de soja para representar os preços dos principais produtos obtidos a partir do processamento do grão.

Entre as variáveis consideradas na análise, a renda é a que exerce maior influência sobre o processamento doméstico de soja, com efeito sempre positivo e transmissão de impacto mais do que proporcional. Outras variáveis muito influenciadas pela renda são as quantidades abatidas de suínos e de aves, com transmissão de impacto próxima da integral. Esses resultados sugerem que a demanda por produtos processados aumenta quando o poder de compra aumenta, seja a demanda por farelo, para o trato de animais, visto que o consumo de carnes também aumenta, seja por óleo, principalmente para a produção de biodiesel, para suprir a frota automotiva do país movida a esse combustível. Tais resultados mostram a importância da renda e o quanto ela impacta o setor. O índice de preços dos derivados de soja também influencia de forma positiva a quantidade de soja processada, mas com transmissão menos do que proporcional, indicando que um aumento no preço dos derivados tende a aumentar a quantidade ofertada. 
Choques não antecipados em abates de suínos e de aves e no preço ao atacado do grão causam efeitos negativos sobre o esmagamento de soja. Esses resultados indicam que à medida que o abate de suínos e de aves aumenta, a demanda por farelo tende a diminuir, no curto prazo, uma vez que a quantidade de animais para alimentar diminui, levando consequentemente a uma queda na demanda por processamento de soja e nos preços de seus derivados. Com a redução da demanda por esmagamento, a oferta de soja em grãos tende a aumentar, com consequente queda do seu preço no mercado interno. Cabe ressaltar que os sinais obtidos para as relações entre o abate de suínos e de aves e o processamento doméstico de soja são coerentes para o curto prazo e para choques não antecipados. Propõe-se, como alternativa, o uso do efetivo de aves e suínos em futuros trabalhos.

Os resultados também mostram que variações no preço ao atacado do grão afetam de forma positiva o índice de preços dos derivados de soja e o preço de exportação do farelo de soja, sugerindo que um aumento no preço da matéria-prima (situação de escassez por quebra de safra, por exemplo) tende a reduzir a oferta de produtos processados e elevar os preços dos derivados. A relevância da renda para explicar mudanças na quantidade de soja processada e nas quantidades abatidas de suínos e de aves é corroborada pela análise da decomposição da variância dos seus erros de previsão.

De modo geral, os resultados do modelo ajustado conseguiram representar de forma conveniente o segmento do mercado de soja de interesse deste artigo, apresentando conclusões que contribuem para melhor entender o comportamento, a dinâmica e a sensibilidade desse setor em determinada conjuntura.

\section{REFERÊNCIAS}

AGÊNCIA NACIONAL DO PETRÓlEO, GÁS NATURAL E BIOCOMBUSTÍVEIS - ANP. Anuário Estatístico 2016. Disponível em: <http:/ / www.anp.gov.br/ ?id=661>. Acesso em: 15 jun. 2016.

ALEXAKIS, C.; BAGNAROSA, G.; DOWLING, M. Do Cointegrated Commodities Bubble Together? The Case of Hog, Corn, and Soybean, 2017. Finance Research Letters, Forthcoming. Disponível em: <https:/ / ssrn.com/abstract=2914152>. Acesso em: 15 ago. 2017.

ALVES, L. R. A. et al. Estrutura de mercado e formação de preços na cadeia produtiva de soja In: BACHA, C. J. C.; ALVES, L. R. A. (Orgs.) Panorama da agricultura brasileira: estrutura de mercado, comercialização, formação de preços, custo de produção e sistemas produtivos. 1 ed. Campinas: Alínea, 2018. p. 95-131.

ARTUZO, F. D. et al. Gestão de custos na produção de milho e soja. Revista Brasileira de Gestão de Negócios, v. 20, n. 2, p. 273-294, 2018. DOI: 10.7819/rbgn.v20i2.3192. 
ASSOCIAÇÃO BRASILEIRA DAS INDÚSTRIAS DE ÓLEOS VEGETAIS ABIOVE. Estatística 2016. Disponível em:<http://www.abiove.org.br/ $>$. Acesso em: 08 jan. 2016.

AVALOS, F. Do oil prices drive food prices? The tale of a structural break. Journal of International Money and Finance, v. 42, p. 253-271, 2014. DOI: 10.1016/j.jimonfin.2013.08.014.

BACCHI, M. R. P. Interdependência dos mercados de gasolina $\mathrm{C}$ e de álcool combustível no Estado de São Paulo. 2007. Tese (Livre-Docência em Ciências) - Escola Superior de Agricultura "Luiz de Queiroz”, Universidade de São Paulo, Piracicaba.

BARROS, G.S.C. Economia da Comercialização Agrícola. Piracicaba: CEPEA/LES-ESALQ/USP, 2012, 245 p.

BARROS, G.S.C.; BACCHI, M.R.P.; BURNQUIST, H.L. Estimação de equações de oferta de exportação de produtos agropecuários para o Brasil (1992/2000). Texto para Discussão 865, IPEA, Brasília, 2002.

BERNANKE, B.S. Alternative explanations of the Money-income correlation. Carnegie-Rochester Conference Series on Public Policy, Rochester, v. 25, p. 49-100, 1986. DOI: 10.3386/w1842.

BINI, D. A. et al. Transmissão de preços ao longo das cadeias produtivas do Brasil. Revista de Economia, Curitiba, v. 42, n. 1, p. 1-20, 2016. DOI: $10.5380 /$ re.v42i1.48660.

BRANDÃO, A. S.; LIMA, E. C. R. Impacts of the U. S. subsidy to soybeans on world prices, production and exports. Revista de Economia e Sociologia Rural, v. 44, n. 4, p. 631-676, 2006. DOI: 10.1590/S0103-20032006000400002.

BRASIL. Ministério de Minas e Energia - MME. Boletim Mensal de Combustíveis Renováveis. Disponível em: <http://www.mme.gov.br/web/guest/publicacoes-e-indicadores>.

Acesso em: 01 ago. 2016.

CEPEA. Centro de Estudos Avançados em Economia Aplicada Esalq/USP. Disponível em: < http://cepea.esalq.usp.br/ >. Acesso em: 20 fev. 2016.

COMPANHIA NACIONAL DE ABASTECIMENTO - CONAB. Séries históricas. 2016. Disponível em: < http://www.conab.gov.br >. Acesso em: 10 fev. 2016.

CORONEL, D. A.; MACHADO, J. A. D.; CARVALHO, F. M. A. Análise da competitividade das exportações do complexo soja brasileiro de 1995 a 2006: uma abordagem de market-share. Revista de Economia Contemporânea, v. 13, n. 2, p. 281-307, 2009. DOI: 10.1590/S1415-98482009000200005. 
DICKEY, D. A.; FULLER, W. A. Distribution of the estimator for autoregressive time series with a unit root. Journal of the American Statistical Association, Alexandria, v. 74, p. 427-431, 1979.

DICKEY, D. A.; FULLER, W. A. Likelihood ratio statistics for autoregressive time series with a unit root. Econometrica, Oxford, v. 49, p. 10571072, 1981. DOI: $10.2307 / 1912517$.

ELLIOTT, D. A.; ROTHENBERG, T. J.; STOCK, J. H. Efficient tests for an autoregressive unit root. Econometrica, Oxford, v.64, n.2, p. 813-836, 1996. DOI: $10.2307 / 2171846$.

ENDERS, W. Applied econometric time series. 4. ed. New Jersey: John Wiley, $2015,480 \mathrm{p}$.

ENGLE, R. F.; GRANGER, C. W. J. Cointegration and error correction: representation, estimation and testing. Econometrica, Oxford, v. 55, n. 2, p. 251-276, 1987. DOI: 10.2307/1913236.

FEISTEL, P.R.; HIDALGO, A.B.; ZUCHETTO, F.B. Determinantes do intercâmbio comercial de produtos agrícolas entre Brasil e China: o caso da soja. Análise Econômica, Porto Alegre, v. 33, n.63, p. 63-89, mar. 2015. Disponível em: <http://seer.ufrgs.br/index.php/analiseeconomica/article/view/42081 >. Acesso em: 15 jul. 2016. DOI: 10.22456/2176-5456.42081.

FIGUEIREDO, P. N. New challenges for public research organisations in agricultural innovation in developing economies: Evidence from Embrapa in Brazil's soybean industry. The Quarterly Review of Economics and Finance, v. 62, p. 21-32, 2016. DOI: 10.1016/j.qref.2016.07.011

FILGUEIRAS, G. C; FERREIRA, M. de N. C.; SANTANA, A; C. de. Análise do mercado e da concentração espacial da cadeia produtiva da soja na Amazônia. Belém: Banco da Amazônia, Estudos Setoriais, v. 4, 2007.

FUNDAÇÃO GETÚTLIO VARGAS - FGV. FGV Dados. Disponível em: <http://portalibre.fgv.br/>. Acesso em: 10 mar. 2016.

GALVÃO, M. C. et al. Influência do preço mundial do petróleo sobre os preços da soja e do açúcar: Uma análise para o Brasil. In: SIMPÓSIO BRASILEIRO DE PESQUISA OPERACIONAL, 47., 2015, Porto de Galinhas. Anais... Porto de Galinhas: Sociedade Brasileira de Pesquisa Operacional, 2015, p. 1193-1204.

GOES, R. H. T. B.; SILVA, L. H. X.; SOUZA, K. A. Alimentos e Alimentação Animal. 1. ed. Dourados: UFGD Editora, 2013, 79 p.

INSTITUTO BRASILEIRO DE GEOGRAFIA E ESTATÍSTICA - IBGE. Sistema IBGE de Recuperação Automática (Sidra). Disponível em: <http://www.ibge.gov.br>. Acesso em: 10 fev. 2016. 
INSTITUTO MATO-GROSSENSE DE ECONOMIA AGROPECUÁRIA IMEA. Entendendo o mercado da soja. 2015. Disponível em: <http://www.imea.com.br/site/projetos.php $>$. Acesso em: 16 jan. 2016.

INSTITUTO DE PESQUISA ECONÔMICA APLICADA - IPEA. Ipeadata. Disponível em: <http:/ / www.ipeadata.gov.br>. Acesso em: 15 mar. 2016.

JOHANSEN, S. Statistical analysis of cointegration vectors. Journal of Economic Dynamics and Control, v. 12, p. 231-254, 1988. DOI: 10.1016/01651889(88)90041-3.

KARALI, B. Do USDA Announcements Affect Comovements Across Commodity Futures Returns? Journal of Agricultural and Resource Economics, v. 37, n. 1, p. 77-97, 2012.

LE CLECH, N. A. Determinantes del precio internacional de la soja. Revista Atlántica de Economía, v. 2, p. 1-22, 2013.

LIU, Q.W. Price relations among hog, corn, and soybean meal futures. The Journal of Futures Markets, v. 25, n. 5, p. 491-514, 2005. DOI: 10.1002/fut.20145.

MACHADO, L. O. Fatores de Formação do Preço da Soja em Goiás. Conjuntura Econômica Goiana, v. 15, p. 45-53, 2010.

NG, S.; PERRON, P. Lag length selection and the construction of unit root tests with good size and power. Econometrica, Oxford, v.69, n. 6, p. 1519$1554,2001$.

PADRÃO, G. A.; GOMES, M. F. M.; GARCIA, J. C. Determinantes Estruturais do Crescimento da Produção Brasileira de Grãos por Estados da Federação: 1989/90/91 e 2006/07/08. Revista Econômica do Nordeste, v. 43, p. 51-66, 2012.

PINTOR, E.; PIACENTI, C. A. Determinantes da expansão da fronteira de produção das culturas de arroz, milho e soja no norte e nordeste brasileiro. Revista Econômica do Nordeste, v. 47, n. 2, p. 41-57, 2016.

SAMPAIO, L. M. B.; SAMPAIO, Y. S. B.; BERTRAND, J. P. Análise da Demanda para Exportações Brasileiras de Soja em grãos e farelo. In: CONGRESSO DA SOCIEDADE BRASILEIRA DE ECONOMIA, ADMINISTRAÇÃO E SOCIOLOGIA RURAL, 42., 2004, Cuiabá. Anais... Cuiabá: Sociedade Brasileira de Economia, Administração e Sociologia Rural, 2004, p. 1-15.

SEDIYAMA, A. F. et al. Análise da estrutura, conduta e desempenho da indústria processadora de soja no Brasil no período de 2003 a 2010. Revista de Economia e Sociologia Rural, Piracicaba - SP, v. 51, n. 1, p. 161-182, 2013. DOI: 10.1590/S0103-20032013000100009. 
SILVA, F.M.; MACHADO, T. Transmissão de preços da soja entre o Brasil e os Estados Unidos no período de 1997 a 2007. Revista Economia e Desenvolvimento, v.1, n. 21, p. 85-103, 2009.

SILVA, M. M.; FRASCAROLI, B.F.; SOBEL, T. F. Transmissão de preços e custos de transação no mercado de soja mato-grossense: uma abordagem por modelos threshold. Revista de Economia e Agronegócio, v. 11, n.2, p. 185210, 2013.

SIMS, C. A. Macroeconomics and reality. Econometrica, Oxford, v. 48, n. 1, p. 1-48, 1980. DOI: 10.2307 / 1912017.

SOUSA, E. P.; CAMPOS, A. C. Transmissão inter-regional de preços no mercado brasileiro de soja. Revista Análise Econômica, v. 27, n.51, p. 83-105, 2009. DOI: $10.22456 / 2176-5456.9701$.

SOUZA M.O. et al. O Complexo da soja: aspectos descritivos e previsões. Pesquisa Operacional para o Desenvolvimento, Rio de Janeiro, v. 2, n. 1, p. 5686, 2010.

SOUZA, M. O.; BACCHI, M. R. P.; ALVES, L. R. A. Determinantes da oferta de exportação brasileira de soja em grãos. In: CONGRESSO DA SOCIEDADE BRASILEIRA DE ECONOMIA, ADMINISTRAÇÃO E SOCIOLOGIA RURAL - SOBER, 56., 2018, Campinas. Anais eletrônicos... Campinas: UNICAMP, 2018. Disponível em: < http:/ / sober.org.br/congresso2018 > . Acesso em: 10 de maio 2019.

SOUZA, S. S. S. et al. Mudanças cambiais e o efeito dos fatores de crescimento das receitas de exportações brasileiras de soja. Revista de Economia e Agronegócio, v. 5, n. 1, p. 1-24, 2007.

TONIN, J. M.; BARCZSZ, S. S. Transmissão de preços da soja entes os mercados externo e interno: uma abordagem para a Região de Maringá. $A$ Economia em Revista, v. 15, p. 35-46, 2007.

UNITED STATES DEPARTMENT OF AGRICULTURE - USDA. Foreign Agricultural Service. World supply and distribution online. 2018. Disponível em: <http://www.fas.usda.gov/psdonline>. Acesso em: 15 mar. 2018. 